\title{
Coupling coefficients and kinetic equation for Rossby waves in multi-layer ocean
}

\author{
T. Soomere \\ Marine Systems Institute at Tallinn Technical University, 1 Paldiski Road, Tallinn EE-10137, Estonia
}

Received: 7 June 2002 - Revised: 17 October 2002 - Accepted: 21 October 2002

\begin{abstract}
The kinetic description of baroclinic Rossby waves in multi-layer model ocean is analysed. Explicit analytical expressions for the coupling coefficients describing energy exchange intensity between different modes are obtained and their main properties are established for the threelayer model. It is demonstrated that several types of interactions vanish in the case of simple vertical structures of the ocean, e.g. when all layers have equal depth. These cases correspond to a zero component of the eigenvectors of the potential vorticity equations. The kinetic equation always possesses a fully barotropic solution. If energy is concentrated in the baroclinic modes, the barotropic mode will necessarily be generated. Motion systems consisting of a superposition of the barotropic and a baroclinic mode always transfer energy to other baroclinic modes.
\end{abstract}

\section{Introduction}

In the framework of weakly non-linear wave theory, natural motion systems are treated as random wave fields with continuous spectral density (spectrum) of energy (e.g. Reznik, 1986; Zakharov et al., 1992; Komen et al., 1994). The wave field evolution is determined by an infinite system of equations with respect to spectral cumulants. Under some restrictions, this system can be reduced to a closed equation for the most important wave field properties. The resulting kinetic equation describes slow (as compared to the characteristic wave period) evolution of the wave system owing to resonant interactions between wave harmonics. Although the assumptions made in deriving of this equation are not perfect (Majda et al., 1997), applications based on the kinetic equation or its simplified versions frequently show excellent and deeply interesting results (e.g. Komen et al., 1994; Zakharov et al., 1992; Zakharov and Pushkarev, 1999).

Correspondence to: $\mathrm{T}$. Soomere

(tarmo@phys.sea.ee)
Rossby waves in a multi-layer ocean serve as an example of medium where several wave classes with different dispersion relations but with comparable frequencies may interact. Interactions of waves from different classes may provide an efficient way of energy redistribution in geophysical flows where frequency domains often are interlapping (e.g. interactions of two inertial waves with a Rossby wave, Wiklund, 1999). The kinetic equation describing interactions of three wave classes was already presented in (Zakharov and Schulman, 1980). However, such systems have been studied in detail only in a particular case when frequencies of the waves are essentially different (Zakharov et al., 1992). In the general case of comparable frequencies and/or a larger number of wave types, the properties of (multi-modal) kinetic equations have not been analysed (Piterbarg, 1998) except for Rossby waves in two-layer medium (Kozlov et al., 1987; Soomere, 1995, 1996).

Barotropic and two-layer models of geophysical flows often inadequately represent the vertical structure of the oceans. In medium latitudes, the seasonal thermocline occasionally creates a three-layer structure. In some areas (e.g. in the Baltic Sea) the barotropic mode is damped and the wave energy is mostly concentrated in the first and the second baroclinic modes. To resolve the vertical structure in such situations, at least three-layer model is necessary.

Application of a model with continuous vertical density alteration (Piterbarg, 1998) is a possibility in numerical simulations. The advantage of the multi-layer model is that the coefficients of the kinetic equation and many basic features of the energy transfer can be found or proved analytically. Also, the important question - whether specific spectral models reproduce the properties of the governing equations - can be clarified to some extent. For example, kinetic models are irreversible and generally possess additional motion invariants. Moreover, their solutions may evolve towards principally different final states as compared to those of the governing equations (cf e.g. results of Carnevale, 1982; Vallis and Maltrud, 1993; Reznik, 1986). One might argue that the kinetic framework is not a proper tool for describing 
evolution of multi-modal Rossby wave systems, because the barotropic mode generally is much faster than the baroclinic ones. This is indeed true, and the analysis below additionally confirms that: a large number of interaction classes involving the barotropic harmonics actually do not redistribute energy, because the corresponding coupling coefficients vanish.

A large part of the analysis is valid for arbitrary wave classes. The detailed derivations have been performed for Rossby waves mostly because they form a convenient and rich in content model system where it is possible to analytically establish many features of the kinetic equations and to interpret the results in a simple physical framework.

The derivation of the kinetic equation in the multi-layer case is a straightforward generalisation of its derivation for the two-layer case (Kozlov et al., 1987). Since an analogous equation has been found in (Piterbarg, 1998) on the basis of Hamiltonian approach and since the interaction coefficients for Rossby waves are well known, the details of the procedure have been omitted.

In order to use the kinetic framework, the equations must first be modified so that each linear part contains one unknown function. Doing this (equivalent to introducing normal modes or diagonalisation of the equations) is accompanied by major changes of the nonlinear parts of the equations. Finally, they consist of certain linear combinations of nonlinear terms from various initial equations. The coefficients at the nonlinear terms we call coupling coefficients because they determine how different modes (wave classes) are coupled with each other. (Notice that, at times, interaction coefficients are also called coupling coefficients, e.g. Axelsson, 1998; the distinguishing is only important in motion systems described by two or more coupled equations). Their appearance depends on the structure of both the linear and the nonlinear part of the governing equations. In the kinetic theory, they form a part of the interaction coefficients (another part of which represents the structure of the nonlinear terms). Generally, coupling coefficients are not rational functions of the coefficients of the initial equations, and they have not been explicitly evaluated in earlier studies (except in the two-layer model; e.g. Kamenkovich et al., 1986; Kozlov et al., 1987).

Since these coefficients first appear at the nonlinear expressions in the equations for the normal modes, their properties are of certain interest in studies into dynamical properties of motions but in the kinetic theory they are particularly important. Namely, they enter into the collision integrals of the kinetic equation and determine the relative role of triads of various types in energy exchange. For example, in the twolayer model of Rossby waves with a proper scaling all types of triads have equal relative energy exchange intensity, except triads containing three baroclinic waves. The intensity of self-interactions of the baroclinic mode crucially depends on the ratio of the depths of the layers and fully ceases in the most simple and widely used case of layers of equal depth (Kozlov et al., 1987; Soomere, 1996). As a consequence, the baroclinic zonal flow and the following large-scale meridional anisotropy do not emerge, and the motion tends to a final state consisting of purely barotropic zonal flow and an isotropic wave system (Soomere, 1995, 1996). Thus, an improper choice of the model may result in a completely different evolution scenario of the whole system. It will be shown below that a number of interactions vanish in a specific case of the three-layer model with equal depths of the topmost and the lowest layers. Such a stratification is not typical in open ocean but frequently occurs in the Baltic Sea (e.g. Aitsam et al., 1984).

The present study focuses on the calculation of the coupling coefficients between different modes or wave classes. The role of the coupling coefficients in the energy exchange as well as their dependence on the particular physical background is analysed in detail. A novel compact representation of multi-layer potential vorticity equation in terms of a combination of bilinear forms is introduced. The analytic expressions for the coefficients of the three-layer model are derived and the dependence of general features of energy exchange on the physical background is analysed. Shown are that certain types of interactions totally vanish in several realistic situations. A new development in the kinetic theory is that particular Rossby wave coupling coefficients in the model in question are shown to vanish if and only if an eigenvector of the governing systems of equations possesses a zero component.

The paper is organised as follows. Section 2 describes decoupling of dynamical equations of large-scale motions in a multi-layer ocean. General expressions for the coupling coefficients are found in Sect. 3. A part of the derivation is fairly general and can be applied for other multi-modal wave systems. The detailed expressions for the coupling coefficients of the Rossby-wave kinetic equation in the three-layer model and analysis of several their properties that directly affect the kinetic equation and its solutions, are presented in Sect. 4. Section 5 contains discussion and Appendix A - the mathematical details of the derivations.

\section{Three-layer model of geostrophic motions and the ki- netic equation}

\subsection{The basic equations}

Dynamics of large-scale motions in the multi-layer ocean with a flat bottom on the $\beta$-plane is described by the potential vorticity equation (Kamenkovich et al., 1986):

$$
\frac{\partial \xi^{(j)}}{\partial t}+\frac{1}{f_{0} \rho_{0}} J\left(p_{j}^{\prime}, \xi^{(j)}\right)=0 ; j=1 \ldots N,
$$

where $N$ is the number of layers, $p_{j}^{\prime}$ is the deviation of the pressure from its equilibrium state in the $j$-th layer,

$$
\xi^{(j)}=\frac{1}{f_{0} \rho_{0}} \Delta p_{j}^{\prime}+\beta y+\frac{f_{0}}{g h_{j}} \frac{p_{j-1}^{\prime}-p_{j}^{\prime}}{\delta \rho_{j}}+\frac{f_{0}}{g h_{j}} \frac{p_{j+1}^{\prime}-p_{j}^{\prime}}{\delta \rho_{j+1}}
$$

is the potential vorticity in the $j$-th layer, $f_{0}$ - the mean value of the Coriolis parameter $f=f_{0}+\beta y, \rho_{0}$ - the mean 


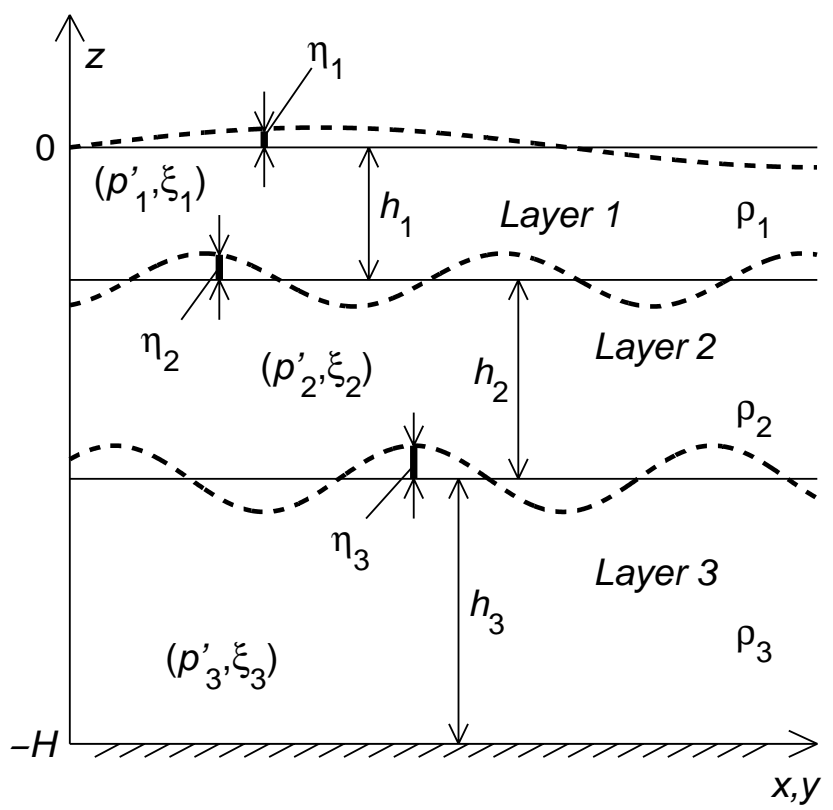

Fig. 1. Three-layer model ocean.

density, $h_{j}, \rho_{j}$ - the mean thickness and density of the $j$ th layer, $J(f, g)=\partial f / \partial x \partial g / \partial y-\partial f / \partial y \partial g / \partial x, \delta \rho_{j}=$ $\rho_{j}-\rho_{j-1} ; 2 \leq j \leq N ; \delta \rho_{1}=\delta \rho_{N+1}=\infty$ and $\delta_{i j}$ is the Kronecker delta. The $x$ - and $y$-axes are directed to the North and the East, respectively and only small deviations from the geostrophic balance are considered. Figure 1 shows vertical structure of the ocean in the three-layer case. In terms of stream functions $\psi_{j}=p_{j}^{\prime}\left(f_{0} \rho_{0}\right)^{-1}$ Eq. (1) reduces to

$$
\begin{aligned}
& \frac{\partial}{\partial t}\left[\Delta \psi_{j}+c_{j}\left(\psi_{j-1}-\psi_{j}\right)+d_{j}\left(\psi_{j+1}-\psi_{j}\right)\right]+\beta \frac{\partial \psi_{j}}{\partial x}+ \\
& +J\left(\psi_{j}, \Delta \psi_{j}\right)+c_{j} J\left(\psi_{j}, \psi_{j-1}-\psi_{j}\right)+ \\
& +d_{j} J\left(\psi_{j}, \psi_{j+1}-\psi_{j}\right)=0, j=1 \ldots N
\end{aligned}
$$

where

$$
\frac{f_{0}^{2} \rho_{0}}{g h_{j} \delta \rho_{j}}=c_{j}, \frac{f_{0}^{2} \rho_{0}}{g h_{j} \delta \rho_{j+1}}=d_{j}, j=1 \ldots N .
$$

It is convenient to write Eq. (2) in the matrix representation

$$
\begin{aligned}
& {\left[\frac{\partial}{\partial t}(\Delta+\mathbf{A})+\beta \frac{\partial}{\partial x}\right] \boldsymbol{\psi}=} \\
& =-\boldsymbol{\psi}^{T} \mathbf{B} J(\cdot, \Delta) \boldsymbol{\psi}-\boldsymbol{\psi}^{T} \mathbf{B} \mathbf{A} J(\cdot, \cdot) \boldsymbol{\psi},
\end{aligned}
$$

where $\boldsymbol{\psi}^{T}=\left(\psi_{1}, \ldots, \psi_{N}\right)$, symbol $(\cdot)^{T}$ means the transposed vector or matrix, $\mathbf{A}$ is the three-diagonal matrix with nonzero elements $c_{j},-c_{j}-d_{j}, d_{j}$ on the $j$-th line, $2 \leq j \leq$ $N ;-d_{1}, d_{1}$ on the first line and $c_{N},-c_{N}$ on the $N$-th line, $\mathbf{B}=\left(\mathbf{B}^{(1)}, \ldots, \mathbf{B}^{(N)}\right)$ is interpreted as a vector, the $j$-th component of which is the $N \times N$-matrix $\mathbf{B}^{(j)}=\left\|\delta_{j m} \delta_{j n}\right\|$ with the only nonzero element $b_{m n}^{j}=1$. The right-hand side of each equation from Eq. (4) can be interpreted as the sum of bilinear forms $\boldsymbol{\psi}^{T} \mathbf{B}^{(j)} J(\cdot, \Delta) \boldsymbol{\psi}$ and $\boldsymbol{\psi}^{T} \mathbf{B}^{(j)} \mathbf{A} J(\cdot, \cdot) \boldsymbol{\psi}$ with $N \times N$-matrices $\mathbf{B}^{(j)}$ and $\mathbf{B}^{(j)} \mathbf{A}$, where the Jacobi operator $J(\cdot, \cdot)$ or the composed operator $J(\cdot, \Delta)$ stands for the multiplication operator.

\subsection{The normal modes}

In order to derive the kinetic equation for wave systems described by Eq. (4), it is necessary to decouple the equations so that the linear part of each equation contains only one unknown function. Formally, doing this is equivalent to introducing normal modes $\left(R_{1}, \ldots, R_{N}\right)=\boldsymbol{R}$ through the substitution

$\boldsymbol{\psi}=\mathbf{S} \boldsymbol{R}, \boldsymbol{\psi}^{T}=\boldsymbol{R}^{T} \mathbf{S}^{T}$,

followed by multiplying the resulting equation from left by $\mathbf{S}^{-1}$. Here $\mathbf{S}$ is a $N \times N$-matrix hereafter called transfer matrix. The procedure (also called the diagonalisation problem) is classical but in systems containing three or more layers the coefficients of the resulting equation generally are not rational functions of the coefficients of Eq. (4) (cf. Piterbarg, 1998).

Substituting Eq. (5) into Eq. (4) results

$$
\begin{aligned}
& {\left[\frac{\partial}{\partial t}(\Delta+\mathbf{A})+\beta \frac{\partial}{\partial x}\right] \mathbf{S} \boldsymbol{R}=} \\
& =-\boldsymbol{R}^{T} \mathbf{S}^{T} \mathbf{B}[j(\cdot, \Delta)+\mathbf{A} J(\cdot, \cdot)] \mathbf{S} \boldsymbol{R} .
\end{aligned}
$$

The matrices $\mathbf{B}^{(j)}$ and $\mathbf{B}^{(j)} \mathbf{A}$ of the bilinear forms have been multiplied by the transfer matrix $\mathbf{S}$ from the right and by its transposed matrix form the left. Eliminating from the $j$ th equation all the $R_{i} ; j \neq i$, is equivalent to multiplying Eq. (6) from left by $\mathbf{S}^{-1}$ and yields

$$
\begin{aligned}
& {\left[\frac{\partial}{\partial t}\left(\Delta+\mathbf{S}^{-1} \mathbf{A S}\right)+\beta \frac{\partial}{\partial x}\right] \boldsymbol{R}=-\mathbf{S}^{-1} \times} \\
& \times\left[\boldsymbol{R}^{T} \mathbf{S}^{T} \mathbf{B S} J(\cdot, \Delta) \boldsymbol{R}+\boldsymbol{R}^{T} \mathbf{S}^{T} \mathbf{B S}\left(\mathbf{S}^{-1} \mathbf{A S}\right) J(\cdot, \cdot) \boldsymbol{R}\right] .
\end{aligned}
$$

The expression in square brackets at the right-hand side of Eq. (7) is a vector, $j$-th component of which is a sum of two bilinear forms. Notice that the matrix multiplication by $\mathbf{S}^{-1}$ does not commute with other operators and consists in summation of the elements of the right-hand side of Eq. (7).

Equation (7) is a solution of the decoupling problem provided $\mathbf{S}^{-1} \mathbf{A S}$ is a diagonal matrix. For any finite $N$, the matrix $\mathbf{A}$ has $N$ different eigenvalues $\lambda_{j} \leq 0$ (Kamenkovich et al., 1986) and $\mathbf{S}^{-1} \mathbf{A S}=\operatorname{diag}\left\|\lambda_{j}\right\|$ indeed is a diagonal matrix provided the column vectors of $\mathbf{S}$ are eigenvectors of $\mathbf{A}$ (equivalently, if $\mathbf{S}$ is the transfer matrix of $\mathbf{A}$ to the normal form). It is convenient to introduce nondimensional variables $R_{j}^{*}, t^{*},\left(x^{*}, y^{*}\right): R_{j}=R_{j}^{*} R_{0} ; t=t^{*} t_{0} ;(x, y)=\left(x^{*}, y^{*}\right) L$, where $t_{0}=(\beta L)^{-1}, R_{0}, L$, are the typical scales of time, the stream function and the horizontal scale, respectively. In terms of the new variables, in Eq. (7) $\lambda_{j}$ are replaced by $\lambda_{j}^{*} L^{-2}$, the factor $\beta$ at the term $\partial \mathbf{R} / \partial x$ disappears and at the right-hand side of Eq. (7) the factor $\varepsilon=R_{0} /\left(\beta L^{3}\right)$ arises. After skipping the star index, we reach:

$$
\begin{aligned}
& \frac{\partial}{\partial t}\left(\Delta R_{j}-a_{j}^{2} R_{j}\right)+\frac{\partial R_{j}}{\partial x}= \\
& =-\varepsilon \sum_{m, n=1}^{N} \gamma_{m n}^{j} J\left(R_{m}, \Delta R_{n}-a_{j}^{2} R_{n}\right), \quad j=1 \ldots N,
\end{aligned}
$$


where $a_{j}=\sqrt{-\lambda_{j}}$ are the nondimensional Rossby radii.

The coefficients $\lambda_{m n}^{j}$ we call coupling coefficients because they describe the intensity of coupling between different modes (wave classes). As different from (Kozlov et al., 1987) where an analogous equation has been derived for two-layer ocean, it turns out that interactions between modes can be described with the use of one set of coefficients $\lambda_{m n}^{j}$. Below it will be demonstrated that, from total $N^{3}$ coefficients, several are identically zero and several always nonzero, but some of them may vanish depending on the vertical structure of the ocean. However, for each mode $j$ there exist nonzero $\lambda_{m n}^{j}, m \neq j, n \neq j$, thus, decoupling of Eq. (8) into independent subsets in the case of Rossby waves does not occur and vanishing of certain coefficients $\lambda_{m n}^{j}$ only leads to minor simplifications of Eq. (8).

\subsection{The multi-wave kinetic equation}

If the parameter $\varepsilon$ in Eq. (8) is small (i.e. the motions are weakly non-linear), it is possible to derive an equation that describes slow temporal evolution of the spectral density (spectrum) of energy of the motions. The derivation procedure is well known (e.g. Benney and Newell, 1969; Reznik, 1984; Kozlov et al., 1987) and we only shortly recall its main points. It is convenient to use Fourier transform $\phi_{j}(\kappa, t)$ of the functions $R_{j}$ defined as

$R_{j}(x, y, t)=\int \phi_{j}(\kappa, t) e^{i(k x+l y)} d \kappa$,

where $\kappa=(k, l)$ is the wave vector and $d \boldsymbol{\kappa}=d k d l$. Substituting Eq. (9) into Eq. (8) yields

$$
\begin{aligned}
& \frac{\partial \phi_{j}}{\partial t}-i \omega_{j} \phi_{j}=\varepsilon \int D_{\boldsymbol{\kappa} \kappa_{1} \kappa_{2}}^{j m n} \phi_{m}\left(\boldsymbol{\kappa}_{1}\right) \phi_{n}\left(\boldsymbol{\kappa}_{2}\right) \times \\
& \times \delta\left(\boldsymbol{\kappa}_{1}+\boldsymbol{\kappa}_{2}-\boldsymbol{\kappa}\right) \delta \boldsymbol{\kappa}_{12}, j=1 \ldots N,
\end{aligned}
$$

where $\omega_{j}=-\beta k /\left(\kappa^{2}+a_{j}^{2}\right)$ is the dispersion relation for the $j$-th mode, $\kappa=|\boldsymbol{\kappa}|, \delta(\kappa)=\delta(k) \delta(l), d \boldsymbol{\kappa}_{12}=d \boldsymbol{\kappa}_{1} d \boldsymbol{\kappa}_{2}$ and

$D_{\kappa \kappa_{1} \kappa_{2}}^{j m n}=\gamma_{m n}^{j} \frac{\left(k_{1} l_{2}-k_{2} l_{1}\right)\left(\kappa_{2}^{2}+a_{n}^{2}-\kappa_{1}^{2}-a_{m}^{2}\right)}{2\left(\kappa^{2}+a_{j}^{2}\right)}$

are the interaction coefficients. For the particular case $j=$ $m=n$ the coupling coefficient $\gamma_{j j}^{j}=1$ is trivial and the coefficients $D_{\kappa \kappa_{1} \kappa_{2}}^{j m n}$ were found already in (Kenyon, 1964; Longuet-Higgins and Gill, 1967). Case $j \neq m=n$ was analysed by Jones (1979). The expression for $D_{\kappa \kappa_{1} \kappa_{2}}^{j m n}$ in Eq. (11) differs from that used in (Reznik, 1984; Kozlov et al., 1987) only in that the indexes $j, m, n$ may now vary from 1 to $N$.

Assume that the functions $R_{j}$ represent homogeneous statistically stationary random fields. Then it is possible to introduce the spectral cumulants (semi-invariants) $F_{j m}(\kappa), \Gamma_{j m n}^{012}\left(\kappa, \kappa_{1}\right), Q_{j m n v}^{0123}\left(\kappa, \kappa_{1}, \kappa_{2}\right)$ etc.:

$<\phi_{j}(\boldsymbol{\kappa}) \phi_{m}\left(\boldsymbol{\kappa}_{1}\right)>=F_{j m}(\boldsymbol{\kappa}) \delta\left(\boldsymbol{\kappa}+\boldsymbol{\kappa}_{1}\right)$,

$$
<\phi_{j}(\kappa) \phi_{m}\left(\kappa_{1}\right) \phi_{n}\left(\kappa_{2}\right)>=\Gamma_{j m n}^{012}\left(\kappa, \kappa_{1}\right) \delta\left(\kappa+\kappa_{1}+\kappa_{2}\right),
$$

$$
\begin{aligned}
& <\phi_{j}(\boldsymbol{\kappa}) \phi_{m}\left(\boldsymbol{\kappa}_{1}\right) \phi_{n}\left(\boldsymbol{\kappa}_{2}\right) \phi_{v}\left(\boldsymbol{\kappa}_{3}\right)>= \\
& =Q_{j m n v}^{0123}\left(\boldsymbol{\kappa}, \boldsymbol{\kappa}_{1}, \boldsymbol{\kappa}_{2}\right) \delta\left(\boldsymbol{\kappa}+\boldsymbol{\kappa}_{1}+\boldsymbol{\kappa}_{2}+\boldsymbol{\kappa}_{3}\right)+ \\
& +F_{j m}(\boldsymbol{\kappa}) \delta\left(\boldsymbol{\kappa}+\boldsymbol{\kappa}_{1}\right) F_{n v}\left(\boldsymbol{\kappa}_{2}\right) \delta\left(\boldsymbol{\kappa}_{2}+\boldsymbol{\kappa}_{3}\right)+ \\
& +F_{j n}(\boldsymbol{\kappa}) \delta\left(\boldsymbol{\kappa}+\boldsymbol{\kappa}_{2}\right) F_{m v}\left(\boldsymbol{\kappa}_{1}\right) \delta\left(\boldsymbol{\kappa}_{1}+\boldsymbol{\kappa}_{3}\right)+ \\
& +F_{j v}(\boldsymbol{\kappa}) \delta\left(\boldsymbol{\kappa}+\boldsymbol{\kappa}_{3}\right) F_{m n}\left(\boldsymbol{\kappa}_{1}\right) \delta\left(\boldsymbol{\kappa}_{1}+\boldsymbol{\kappa}_{2}\right) \text { etc. }
\end{aligned}
$$

The semiinvariant $F_{j j}(\boldsymbol{\kappa})$ has the meaning of spectral density of energy of the $j$-th mode. With the use of Eq. (10), it is straightforward to obtain a coupled infinite system of equations for the sequence of semi-invariants (12.1-3). The simplest way to close this system consists in neglecting the fourth-order and the higher order cumulants (Hasselmann, 1962; later on Reznik, 1984; showed that much weaker closure hypothesis may be used). Asymptotic analysis of the system of equations for the cumulants $F_{j m}, \Gamma_{j m n}^{012}\left(\kappa, \kappa_{1}\right)$, is described in detail e.g. in (Kenyon 1964; Benney and Newell, 1969; Reznik, 1984). Analysis of the main points of the derivation and the validity of the underlying approximations is presented in (Majda et al., 1997). The quite tedious analysis is omitted here, because it contains nothing instructive. The following system of equations (multi-modal or multi-wave kinetic equation) with respect to the spectral density of energy (spectrum) of the $j$-th mode $F_{j}=F_{j}(\kappa, \tau)$ (the double index ${ }^{\prime} j j^{\prime}$ has been replaced by the single one) can be obtained:

$$
\begin{aligned}
\frac{\partial F_{j}}{\partial \tau} & =4 \pi \sum_{m, n=1}^{N} I_{j m n}, j=1 \ldots N ; \\
I_{j m n} & =\int D_{\kappa \kappa_{1} \kappa_{2}}^{j m n} K_{j m n} \delta\left(\omega_{j m n}^{012}\right) \delta\left(\kappa_{012}\right) d \kappa_{12} .
\end{aligned}
$$

Here $\tau=\varepsilon^{2} t$ is slow time, $I_{p m n}$ are the collision integrals describing energy exchange between harmonics belonging to the modes with numbers $j, m, n$, the integrals are taken over the four-dimensional space $\boldsymbol{R}^{2}\left(\boldsymbol{\kappa}_{1}\right) \times \boldsymbol{R}^{2}\left(\boldsymbol{\kappa}_{2}\right)$, $\omega_{j m n}^{012}=\omega_{j}(\boldsymbol{\kappa})+\omega_{m}\left(\boldsymbol{\kappa}_{1}\right)+\omega_{n}\left(\boldsymbol{\kappa}_{2}\right), \boldsymbol{\kappa}_{012}=\boldsymbol{\kappa}+\boldsymbol{\kappa}_{1}+\boldsymbol{\kappa}_{2}$ and

$$
K_{j m n}=D_{\kappa_{1} \kappa_{2}}^{j m n} F_{m}\left(\kappa_{1}\right) F_{n}\left(\kappa_{2}\right)+
$$$$
+D_{\boldsymbol{\kappa}_{1} \kappa_{2} \kappa}^{m n j} F_{n}\left(\boldsymbol{\kappa}_{2}\right) F_{j}(\boldsymbol{\kappa})+D_{\boldsymbol{\kappa}_{2} \kappa \kappa_{1}}^{n j m} F_{j}(\boldsymbol{\kappa}) F_{m}\left(\boldsymbol{\kappa}_{1}\right) \text {. }
$$

The derivation is valid for any set of wave classes possessing triad interactions provided the double resonance does not occur (Reznik, 1984). The sign indices in Eqs. $(13,14)$ are avoided through allowing negative frequencies of Rossby waves with $k>0$. Equation (13) is equivalent to the analogical equation with respect to wave action derived on the basis of the Hamiltonian approach (Piterbarg, 1998). The proof of basic properties of the Eq. (13) such as conservation of energy and wave momentum, and the $H$-theorem, is straightforward.

\section{The coupling coefficients}

The coupling coefficients $\gamma_{m n}^{j}$ form a part of dynamical equations (8) for the normal modes. They explicitly depend on the 
vertical structure of the model (but are independent of properties of particular wave harmonics) and control general energy exchange intensity between and within the modes. The remainder of the coefficients $D_{\kappa \kappa_{1} \kappa_{2}}^{j m n}$ represents the structure of nonlinear terms of Eq. (8) and describes energy exchange details in particular triads. Obviously, in wave systems containing one type of waves only, distinguishing of coupling coefficients is unessential.

The coupling coefficients can be found a straightforward manner from Eq. (7). Let us use the traditional notation for line $\boldsymbol{s}_{i}^{T}=\left\|s_{i n}\right\|, 1 \leq n \leq N$, and column vectors $\boldsymbol{s}^{j}=\left\|s_{m j}\right\|, 1 \leq m \leq N$, for the transfer matrix $\mathbf{S}=\left\|s_{i j}\right\|$. The vectors $\boldsymbol{s}^{j}$ are defined as $\left(\mathbf{A}-\lambda_{j} \mathbf{I}\right) \boldsymbol{s}^{j}=\mathbf{0}, 1 \leq j \leq N$, where $\mathbf{I}$ is the unity matrix. The product $\mathbf{B}^{(i)} \mathbf{S}$ is a matrix in which the $i$-th line vector is $\boldsymbol{s}_{i}^{T}$ and all other entries are zeros. Multiplying $\mathbf{B}^{(i)} \mathbf{S}$ from left by $\mathbf{S}^{T}$ gives

$\mathbf{S}^{T} \mathbf{B}^{(i)} \mathbf{S}=\left\|\begin{array}{c}s_{i 1} \mathbf{s}_{i}^{T} \\ \ldots \\ s_{i N} \mathbf{S}_{i}^{T}\end{array}\right\|=\left\|s_{i 1} \mathbf{S}_{i} \ldots s_{i N} \mathbf{S}_{i}\right\|$.

The remaining multiplication in Eq. (7) from left by $\mathbf{S}^{-1}$ leads finally to

$\left\|\gamma_{m n}^{j}\right\|=\sum_{i=1}^{N} \hat{s}_{j i} \mathbf{S}^{T} \mathbf{B}^{(i)} \mathbf{S}$

where $\hat{s}_{m n}$ are the elements of the matrix $\mathbf{S}^{-1}$. From Eq. (15) it follows that a coefficient $\gamma_{m n}^{j}$ is the scalar product of the column vectors $\boldsymbol{s}_{m}^{T}$ and $\boldsymbol{s}_{n}^{T}$ under the metric defined by the $j$-th line vector $\hat{\boldsymbol{s}}_{j}$ of the matrix $\mathbf{S}^{-1}$ :

$\gamma_{m n}^{j}=\sum_{i=1}^{N} \hat{s}_{j i} s_{i m} s_{i n}, j, m, n=1 \ldots N$.

Direct evaluation of the coupling coefficients is quite tedious even in the simplest case of the two-layer model. However, there exist several general properties of these coefficients. First notice that $\left(\mathbf{S}^{T} \mathbf{B}^{(i)} \mathbf{S}\right)^{T}=\mathbf{S}^{T} \mathbf{B}^{(i)} \mathbf{S}$, consequently, all the resulting matrices in Eq. (15) are symmetric and

$\gamma_{m n}^{j}=\gamma_{n m}^{j}, j, m, n=1 \ldots N$.

A specific property of the coefficients $\gamma_{m n}^{j}$ in the case of wave systems possessing a zero eigenvalue $\lambda_{1}=0$ allows to simply evaluate several coupling coefficients between the first and the higher modes. It is easy to establish that the coordinates of the eigenvector $s^{1}$ corresponding to $\lambda_{1}=0$ are equal, e.g. $s_{i 1}=a$. Denoting the cofactor of $s_{i j}$ as $A_{i j}$, we have:

$$
\begin{aligned}
& \gamma_{1 j}^{j}=\sum_{i=1}^{N} \hat{s}_{j 1} s_{i 1} s_{i j}=a \sum_{i=1}^{N} \hat{s}_{j 1} s_{i j}= \\
& =a|\mathbf{S}|^{-1} \sum_{i=1}^{N} A_{j 1} s_{i j}=a, j=1 \ldots N
\end{aligned}
$$

since $\sum_{i=1}^{N} A_{j 1} s_{i j}$ is the determinant $|\mathbf{S}|$, expressed in terms of the elements of the $j$-th line of $\mathbf{S}$ and the corresponding cofactors.

A specific property of Rossby waves is that the eigenvectors of $\mathbf{A}$ (columns of the transfer matrix $\mathbf{S}$ ) are orthogonal with respect to the vector $\boldsymbol{h}=\left(h_{1}, h_{2}, \ldots, h_{N}\right)$, where $h_{i}$ is the thickness of the $i$-th layer (Kamenkovich et al., 1986). This property yields

$\gamma_{12}^{1}=\gamma_{13}^{1}=\gamma_{23}^{1}=0$.

The Hamiltonian structure of baroclinic Rossby wave systems yields that the (Jacobi) identities

$$
\begin{aligned}
& \frac{D_{\boldsymbol{k} \kappa_{1} \kappa_{2}}^{j m n}}{\gamma_{m n}^{j} \omega_{j}(\kappa)}=\frac{D_{\boldsymbol{\kappa}_{1} \kappa_{2} \kappa}^{m n j}}{\gamma_{n j}^{m} \omega_{m}\left(\boldsymbol{\kappa}_{1}\right)}=\frac{D_{\boldsymbol{\kappa}_{2} \kappa \kappa_{1}}^{n j m}}{\gamma_{j m}^{n} \omega_{n}\left(\kappa_{2}\right)}= \\
& =V_{\boldsymbol{\kappa} \kappa_{1} \kappa_{2}}^{j m n}, j, m, n=1 \ldots N,
\end{aligned}
$$

are satisfied at the resonance curves $\omega_{j m n}^{012}=0, \kappa_{012}=0$. The quantity $V_{\boldsymbol{k} \kappa_{1} \kappa_{2}}^{j m n}$ is called the matrix element of the wave system. (Studies of the kinetic equation based on Hamiltonian approach (e.g. Balk et al., 1990; Zakharov et al., 1992, among others) ascertain these features proceeding from the conservation laws of the corresponding weakly non-linear systems. For Rossby wave systems it is traditional to obtain them in a straightforward way; Piterbarg, 1998). Equations (20) yield that the coupling coefficients are invariant with respect to cyclic permutations $j \rightarrow m \rightarrow n \rightarrow j$ of the mode numbers, i.e. that

$\gamma_{m n}^{j}=\gamma_{n j}^{m}=\gamma_{j m}^{n}, j, m, n=1 \ldots N$.

(Notice that this property for nonzero coefficients only holds if lengths of the eigenvectors of $\mathbf{A}$ are chosen properly; see below.). In particular, this property together with Eq. (17) results that $\gamma_{11}^{2}=\gamma_{11}^{3}=\gamma_{12}^{3}=0$. Physically, it means that in the three-layer ocean model only two classes of interactions involving the barotropic mode are energetically important: triads of barotropic waves and triads consisting of one barotropic and two baroclinic waves from a fixed mode. All other interactions involving barotropic components vanish. Therefore, within the three-layer model there is no energy exchange within triplets representing three different modes. The physical reason is that all such interactions are of the order of the vertical density alteration (Kozlov et al., 1987).

\section{The analytical expressions for the coupling coeffi- cients}

4.1 The coupling coefficients for the two-layer and the three-layer models

In the two-layer case the eigenvalues $\lambda_{1}=0, \lambda_{2}=-h_{1}^{-1}-$ $h_{2}^{-1}$ can be explicitly calculated (Appendix A) and the coupling coefficients are

$\left\|\gamma_{m n}^{1}\right\|=\left\|\begin{array}{cc}a & 0 \\ 0 & \frac{b^{2} h_{1} h_{2}}{a}\end{array}\right\|,\left\|\gamma_{m n}^{2}\right\|=\left\|\begin{array}{cc}0 & a \\ a & b\left(h_{2}-h_{1}\right)\end{array}\right\|$, 
where $a, b$ are arbitrary nonzero coefficients. (Notice that a particular scaling does not change general interaction properties and basically reduces to changes of the slow time scale.) The coefficients $\gamma_{m n}^{j}$ coincide with those used in (Kozlov et al., 1987, Soomere, 1996) if

$a=\frac{h_{1}+h_{2}}{h_{1}}, b=\frac{h_{1}+h_{2}}{h_{1}^{2}}$.

The most convenient scaling is

$a=1, b=1 / \sqrt{h_{1} h_{2}}$,

that yields $\gamma_{11}^{1}=\gamma_{22}^{1}=\gamma_{12}^{2}=\gamma_{21}^{2}=1, \gamma_{22}^{2}=\sqrt{h_{2} / h_{1}}-$ $\sqrt{h_{1} / h_{2}}$. The advantage of this scaling is that it explicitly expresses the invariance of the dynamics of the two-layer model with respect to the numbering of the layers. Indeed, the kinetic equation does not change if the transposition $h_{1} \leftrightarrow h_{2}$ is made because only products of coupling coefficients enter into each collision integral.

The expressions for coupling coefficients are much more complex in the case of the three-layer model because generally they cannot be expressed as rational functions of the coefficients of the governing Eqs. (4). The matrix $\mathbf{A}$ in Eq. (5) has a zero eigenvalue $\lambda_{1}=0$ whereas other eigenvalues satisfy the characteristic equation

$\lambda^{2}+\lambda\left(d_{1}+d_{2}+c_{2}+c_{3}\right)+d_{1} d_{2}+d_{1} c_{3}+c_{2} c_{3}=0$.

Equation (23) generally does not have rational eigenvalues except in the case $c_{1}=d_{3}$ (including the simplest case when all the layers have equal depths) when

$\lambda_{2}=-d_{1}, \quad \lambda_{3}=-d_{2}-c_{2}-c_{3}=\lambda_{2}-d_{2}-c_{2}$.

The solutions of Eq. (23) are invariant with respect to the transposition $\left(d_{1}, d_{2}\right) \leftrightarrow\left(c_{2}, c_{3}\right)$ that causes only $\lambda_{2} \leftrightarrow \lambda_{3}$ and is equivalent to reversing of the counting order of the layers. Thus, the coupling coefficients are independent on the direction of counting the layers. This property is a generalisation of the fact that the two-layer kinetic equation is invariant with respect to the transposition $h_{1} \leftrightarrow h_{2}$.

In the general case, the transfer matrix and its inverse are given by Eq. (A7) and the nontrivial coupling coefficients are (see Appendix A for details):

$$
\begin{aligned}
& \gamma_{11}^{1}=a, \gamma_{22}^{1}=\frac{b^{2}\left(\lambda_{2}-\lambda_{3}\right)\left(\lambda_{2}+d_{1}\right) c_{3}}{a \lambda_{3}\left(\lambda_{2}+c_{3}\right) d_{1}}, \\
& \gamma_{33}^{1}=\frac{c^{2}\left(\lambda_{3}-\lambda_{2}\right)\left(\lambda_{3}+d_{1}\right) c_{3}}{a \lambda_{2}\left(\lambda_{3}+c_{3}\right) d_{1}}
\end{aligned}
$$

$\gamma_{12}^{2}=\gamma_{21}^{2}=a$,

$\gamma_{22}^{2}=b\left[\frac{c_{3}\left(\lambda_{2}+d_{1}\right)}{d_{1}\left(\lambda_{2}+c_{3}\right)}+1-\frac{\lambda_{2}\left(\lambda_{2}+d_{1}\right)}{\left(\lambda_{3}-\lambda_{2}\right) d_{1}}\right]$,

$\gamma_{23}^{2}=\gamma_{32}^{2}=c \frac{\lambda_{2}\left(\lambda_{3}+d_{1}\right)}{\left(\lambda_{2}-\lambda_{3}\right) d_{1}}$,

$\gamma_{33}^{2}=\frac{c^{2} \lambda_{3}^{2}\left(\lambda_{3}+d_{1}\right)\left(\lambda_{2}+c_{3}\right)}{b \lambda_{2}\left(\lambda_{3}-\lambda_{2}\right)\left(\lambda_{3}+c_{3}\right) d_{1}}$, $\gamma_{13}^{3}=\gamma_{31}^{3}=a, \gamma_{22}^{3}=\frac{b^{2} \lambda_{2}^{2}\left(\lambda_{2}+d_{1}\right)\left(\lambda_{3}+c_{3}\right)}{c \lambda_{3}\left(\lambda_{3}-\lambda_{2}\right) d_{1}\left(\lambda_{2}+c_{3}\right)}$,

$\gamma_{23}^{3}=\gamma_{32}^{3}=b \frac{\lambda_{3}\left(\lambda_{2}+d_{1}\right)}{\left(\lambda_{3}-\lambda_{2}\right) d_{1}}$,

$\gamma_{33}^{3}=c\left[\frac{\lambda_{3}\left(\lambda_{3}+d_{1}\right)}{\left(\lambda_{2}-\lambda_{3}\right) d_{1}}-\frac{c_{3}\left(\lambda_{3}+d_{1}\right)}{d_{1}\left(\lambda_{3}+c_{3}\right)}-1\right]$.

Equations (25)-(27) explicitly demonstrate that the property $\gamma_{m n}^{j}=\gamma_{n j}^{m}=\gamma_{j m}^{n}$ requires a specific adjustment of the lengths of the eigenvectors of $\mathbf{A}$.

4.2 Equal reduced depth of the topmost and the lowest layers

The coupling coefficients of the two-layer model in Eq. (21) are always well defined. The transfer matrix in the case of the three-layer model is regular in the physically meaningful cases when all the layer depths are finite and nonzero. The expressions for the coupling coefficients in Eqs. (25)-(27) may be improper if and only if the product $\left(\lambda_{2}+d_{1}\right)\left(\lambda_{3}+\right.$ $\left.d_{1}\right) \times \times\left(\lambda_{2}+c_{3}\right)\left(\lambda_{3}+c_{3}\right)=0$. Since $\lambda_{3} \neq \lambda_{2}$, this may happen if and only if one of the components of the eigenvectors $s^{2}$ or $s^{3}$ is zero.

If $\lambda_{2}+d_{1}=0$ or $\lambda_{2}+c_{3}=0$, Eq. (A3) yield $s_{2}=0$. From the characteristic equation (23) it follows that $\lambda_{3}=$ $-c_{2}-c_{3}-d_{2}$ or $\lambda_{3}=-d_{1}-d_{2}-c_{2}$. Since $c_{2} \neq 0$, this is possible only if $d_{1}=c_{3}$ and $\lambda_{3}+d_{1}=\lambda_{3}+c_{3}=-d_{2}-c_{2} \neq 0$. The case $\left(\lambda_{3}+d_{1}\right)\left(\lambda_{3}+c_{3}\right)=0$ also yields $d_{1}=c_{3}$ but gives $\lambda_{2}=-d_{1}-d_{2}-c_{2}<\lambda_{3}$ and is implicitly excluded in the traditional approach, because it is assumed that $\lambda_{i}<\lambda_{j}$ provided $i>j$. Thus, expressions (25)-(27) may fail if and only if $d_{1}=c_{3}$, or in models where the reduced depths $h_{1} \delta \rho_{2}$ and $h_{3} \delta \rho_{3}$ of the uppermost and the lowermost layers are equal. This case includes the simplest three-layer model with equal depths and equal density differences between layers. In realistic problems, such situation may occur either in atmospheric dynamics or in studies into motions in relatively shallow strongly stratified basins like the Baltic Sea.

As for the coupling coefficients, notice first that Eqs. (16)(19) are valid for any combination of the parameters of the original Eqs. (2) and (4). The expressions for the remaining coefficients simplify greatly and can be obtained from Eqs. (16) and (A18) in a straightforward manner:

$$
\begin{aligned}
& \gamma_{11}^{1}=a, \gamma_{22}^{1}=\frac{b^{2}\left(d_{2}+c_{2}\right) c_{2}}{a\left(d_{1}+d_{2}+c_{2}\right) d_{2}}, \\
& \gamma_{33}^{1}=\frac{c^{2}\left(d_{2}+c_{2}\right)}{a d_{1}}, \\
& \gamma_{12}^{2}=\gamma_{21}^{2}=a, \gamma_{22}^{2}=b \frac{d_{2}-c_{2}}{d_{2}}, \\
& \gamma_{23}^{2}=\gamma_{32}^{2}=c, \gamma_{33}^{2}=0, \\
& \gamma_{13}^{3}=\gamma_{31}^{3}=a, \gamma_{22}^{3}=\frac{b^{2} d_{1} c_{2}}{c\left(d_{1}+d_{2}+c_{2}\right) d_{2}}, \\
& \gamma_{23}^{3}=\gamma_{32}^{3}=0, \gamma_{33}^{3}=c \frac{d_{1}-d_{2}-c_{2}}{d_{1}} .
\end{aligned}
$$




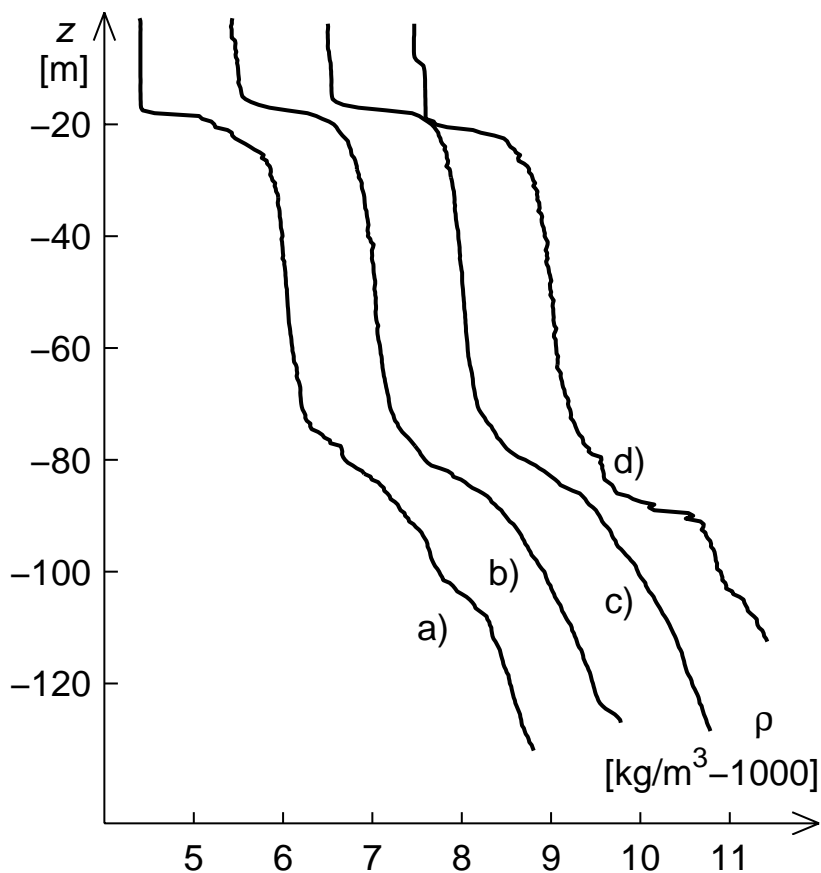

Fig. 2. Vertical density profiles in the Gotland Basin, Baltic Sea, 26.-28.08.1986: (a) $57^{\circ} 31,6^{\prime} \mathrm{N}, 20^{\circ} 34,2^{\prime} \mathrm{E}$; (b) $57^{\circ} 37,0^{\prime} \mathrm{N}$, $20^{\circ} 32,4^{\prime} \mathrm{E}$; (c) $57^{\circ} 38,5^{\prime} \mathrm{N}, 20^{\circ} 36,1^{\prime} \mathrm{E}$; (d) $57^{\circ} 38,0^{\prime} \mathrm{N}, 20^{\circ} 42,5^{\prime} \mathrm{E}$. The distance from the end of a profile to the bottom is about $10 \mathrm{~m}$. The total density alteration about $0.5 \%$ is typical for the Baltic Sea. The profiles (b), (c), (d) are shifted to the right by 1, 2 or 3 density units, respectively.

The performed analysis reveals that there are no other cases when the derived expressions for the coupling coefficients might fail.

4.3 Vanishing coupling coefficients and eigenvector components

In the case of two-layer model the energy transfer within the baroclinic mode ceases in the frequently used particular case $h_{1}=h_{2}$ (Kozlov et al., 1987; Soomere, 1996). This happens exclusively owing to the fact that the corresponding coupling coefficient vanishes and leads to a specific situation where energy redistribution within the baroclinic mode is governed exclusively by intermodal interactions that do not support baroclinic zonal flow (Soomere, 1995, 1996).

Within the three-layer model the conditions for vanishing certain coupling coefficients are more complex. The analysis in Sect. 3 demonstrates that the coefficients $\gamma_{11}^{1}, \gamma_{12}^{2}$ and $\gamma_{13}^{3}$ are always nonzero. From Eq. (25) it follows that $\gamma_{22}^{1}$ and $\gamma_{33}^{1}$ might vanish provided $\lambda_{j}+d_{1}=0$ but Eq. (28) confirm that they are always nonzero. This is not surprising because otherwise the evolution of the barotropic mode would be not coupled with the other modes.

The coefficients $\gamma_{23}^{2}, \gamma_{33}^{2}$ and $\gamma_{23}^{3}$ also may vanish only if $\lambda_{j}+d_{1}=0, j=2,3$. The above has shown that indeed the coefficients $\gamma_{33}^{2}=\gamma_{23}^{3}=0$ provided $d_{1}=c_{3}$, i.e. in the models with equal reduced depths of the uppermost and the lowest layers. In such models, there is no energy exchange in triplets containing one wave from the first baroclinic mode and two waves from the second baroclinic mode. There still exists energy exchange between the baroclinic modes in triads containing two waves from the first baroclinic mode and one from the second mode, because $\gamma_{23}^{2}=c$ and the coefficient $\gamma_{23}^{2}$ never vanishes. The coefficient $\gamma_{22}^{3}$ may vanish provided $\left(\lambda_{3}+d_{2}+c_{3}\right)\left(\lambda_{3}+c_{3}\right)=0$. The case $\lambda_{3}+c_{3}=0$ yields $d_{1}=c_{3}$ when $\gamma_{22}^{3}$ is definitely nonzero. The other condition $\lambda_{3}+d_{2}+c_{3}=0$ is equivalent to $\lambda_{2}+d_{1}+c_{2}=0$ and yields $d_{2} c_{2}=0$ that is impossible. Thus, $\gamma_{22}^{3}$ never vanishes.

The modal structure of the flow can be found from Eq. (5) as $\boldsymbol{R}=\mathbf{S}^{-1} \boldsymbol{\psi}$; in the particular case $d_{1}=c_{3}$ it can be expressed with the use of Eq. (A18) as $R_{1} \sim c_{2} \psi_{1}+d_{1} \psi_{2}+$ $d_{2} \psi_{3}, R_{2} \sim \psi_{1}-\psi_{3}, R_{3} \sim c_{2} \psi_{1}-\left(c_{2}+d_{2}\right) \psi_{2}+d_{2} \psi_{3}$. Notice that motions in the intermediate layer do not enter into the equation for the first baroclinic mode whereas its structure does not depend on the depth of this layer. This equation is perfectly symmetric with respect to motions in the topmost and the lowest layers.

The coefficients $\gamma_{22}^{2}$ and $\gamma_{33}^{2}$ may vanish for certain combinations of the parameters of Eq. (2). However, we were not able to find a simple and exhaustive analytic criterion for these coefficients to vanish. With the use of the characteristic Eq. (23) it is possible to exclude from the conditions $\gamma_{22}^{2}=0$ and $\gamma_{33}^{2}=0$ all cubic and quadratic terms with respect to the eigenvalues $\lambda_{2}$ and $\lambda_{3}$, and to reduce these conditions to $\lambda_{2}=G\left(d_{1}, d_{2}, c_{2}, c_{3}\right)$, where $G$ is a rational function with respect to all its arguments. Therefore, the coefficients $\gamma_{22}^{2}$ and $\gamma_{33}^{2}$ only may vanish provided Eq. (23) has rational solutions. It has been shown in Sect. 4.1 that the solutions indeed are rational in the case $d_{1}=c_{3}$ but there may exist other solutions.

From Eq. (26) it follows that the coefficient $\gamma_{22}^{2}$ vanishes exactly and energy exchange within the first barotropic mode is idle if also $d_{2}=c_{2}$. This condition means that $\delta \rho_{2}=\delta \rho_{3}$ (the changes in density are equal and, consequently, $h_{1}=h_{3}$ ) whereas the spectral evolution of the first baroclinic mode is fully governed by interactions with the other modes. The structure of the first baroclinic mode does not change but the barotropic $R_{1} \sim \psi_{1}+h_{2} \psi_{2} / h_{1}+\psi_{3}$ and the second baroclinic mode $R_{3} \sim \psi_{1}-2 \psi_{2}+\psi_{3}$ contain an equal portion of the motions in the topmost and the lowest layers.

Energy exchange within the second baroclinic mode is idle (equivalently, $\gamma_{33}^{3}=0$ ) on condition that $d_{1}=d_{2}+c_{2}$ or, in the case in question, $h_{2}=h_{1}+h_{3}$. The barotropic $R_{1} \sim$ $\delta \rho_{3} \psi_{1}+\left(\delta \rho_{2}+\delta \rho_{3}\right) \psi_{2}+\delta \rho_{2} \psi_{3}$ and the second baroclinic mode $R_{3} \sim \delta \rho_{3} \psi_{1}-\left(\delta \rho_{2}+\delta \rho_{3}\right) \psi_{2}+\delta \rho_{2} \psi_{3}$ again contain an equal portion of the motions in the topmost and the lowest layers.

Further, in the particular case $\delta \rho_{2}=\delta \rho_{3}, h_{1}=h_{3}, h_{2}=$ $2 h_{1}$, interactions within both the baroclinic modes do not cause actual energy redistribution. The modal structure is particularly simple: $R_{1} \sim \psi_{1}+2 \psi_{2}+\psi_{3}, R_{3} \sim \psi_{1}-2 \psi_{2}+$ $\psi_{3}$. Thus, the rather realistic case when the density changes 
between layers are equal, depths of the lower and the upper layers are equal, and the intermediate layer is twice as thick as the other layers, serves as "generalisation" of the classical two-layer model with equal depths where self-interactions of the baroclinic mode are idle. Such a vertical stratification seldom occurs in the open ocean (where typically the depth of the lowest layer greatly exceeds that of the other ones) but frequently appears in the Baltic Sea. The density structure of its the central part often consists of a mixed upper layer, well-defined seasonal thermocline at a depth about 20-40 m, an intermediate layer and main halocline at a depth about $80-100 \mathrm{~m}$ whereas the total depth is about $120-140 \mathrm{~m}$ (Fig. 2) and density changes between the layers are more or less equal.

From Eqs. (A5), (A18) it follows that in the particular case $\lambda_{j}+d_{1}=0$ (i.e. when several coupling coefficients vanish from those that normally are nonzero, see Table 1) the eigenvector(s) possess zero components. Further, it can be shown that maximally one of the eigenvector components (equivalently, only one of the entries of matrix $\mathbf{S}$ or its inverse $\mathbf{S}^{-1}$ ) may vanish, and that exactly one component vanishes if an additional coupling coefficient is zero. This observation indicates that vanishing coupling may occur solely if eigenvectors of $\mathbf{A}$ have a zero entry. This property allows to detect specific flow regimes (that correspond to vanishing of certain types of interactions) directly from the analysis of eigenvectors of the governing Eqs. (2) or (4).

\subsection{The convenient scalings}

In the derivations above there are three "free" parameters (the lengths of the eigenvectors) that can be chosen in many different ways. Traditionally, transfer to the normal modes in Eq. (4) is given in the form $R_{j}=\psi_{1}+\ldots$, i.e. through the matrix $\mathbf{S}$, the first column of the inverse matrix of which is a unity vector (Kozlov et al., 1987; Kamenkovich, 1986). From the analysis above it becomes clear that it is always possible to choose the lengths of the eigenvectors of $S$ (equivalently, the parameters $a, b, c$ ) so that this representation can be used. However, this scaling leads to many nontrivial (i.e. other than zero or unity) coupling coefficients at the collision integrals and requests the baroclinic mode to be rescaled in the energy conservation law (Kozlov et al., 1987).

The most convenient scaling apparently is the one that leads to maximally simple form of the coupling coefficients. A possibility is to choose the parameters $a, b, c$ so that as many as possible coefficients $\gamma_{m n}^{j}=1$. The analysis of the two-layer model and Eq. (22) suggests the scaling

$$
\begin{aligned}
& a=1, b^{2}=\frac{\lambda_{3}\left(\lambda_{2}+c_{3}\right) d_{1}}{\left(\lambda_{2}-\lambda_{3}\right)\left(\lambda_{2}+d_{1}\right) c_{3}}, \\
& c^{2}=\frac{\lambda_{2}\left(\lambda_{3}+c_{3}\right) d_{1}}{\left(\lambda_{3}-\lambda_{2}\right)\left(\lambda_{3}+d_{1}\right) c_{3}}
\end{aligned}
$$

that results in $\left\|\gamma_{m n}^{1}\right\|=\mathbf{I}$ and

$$
\gamma_{23}^{2}=\gamma_{22}^{3}=\frac{\lambda_{3}}{\lambda_{3}-\lambda_{2}} \sqrt{\frac{\lambda_{3}\left(\lambda_{3}+c_{3}\right)\left(\lambda_{3}+d_{1}\right)}{\left(\lambda_{3}-\lambda_{2}\right) c_{3} d_{1}}},
$$

$\gamma_{33}^{2}=\gamma_{23}^{4}=\frac{\lambda_{3}}{\lambda_{2}-\lambda_{3}} \sqrt{\frac{\lambda_{3}\left(\lambda_{2}+c_{3}\right)\left(\lambda_{2}+d_{1}\right)}{\left(\lambda_{2}-\lambda_{3}\right) c_{3} d_{1}}}$.

The expressions for $\gamma_{22}^{2}$ and $\gamma_{33}^{3}$ remain quite tedious. The advantage of this scaling is that the coupling coefficients $\gamma_{m n}^{j}$ are explicitly invariant with respect to cyclic permutation $j \rightarrow m \rightarrow n \rightarrow j$ of the mode numbers. Its another important benefit is that simply the sum of energies of the modes enters into the energy conservation law.

\subsection{Specific flow regimes}

Several interesting properties of the multi-modal kinetic equation (13) become evident directly from the analysis of the coupling coefficients. The fact that $\gamma_{22}^{1}$ and $\gamma_{33}^{1}$ are nonzero means that the barotropic mode, if not present initially, will always be generated. Indeed, if at some time moment $\tau=\tau_{0}$ the barotropic energy $F_{1}=0$, then

$$
\begin{aligned}
& \frac{\partial F_{1}\left(\tau_{0}\right)}{\partial \tau}=\pi \sum_{j=2,3} \int\left(\gamma_{j j}^{1} C_{\kappa \kappa k_{1} \kappa_{2}}^{1 j j}\right)^{2} F_{j}\left(\boldsymbol{\kappa}_{1}\right) F_{j}\left(\boldsymbol{\kappa}_{2}\right) \times \\
& \times \delta\left(\omega_{1 j j}^{012}\right) \delta\left(\kappa_{012}\right) d \boldsymbol{\kappa}_{12} \geq 0,
\end{aligned}
$$

where

$$
C_{\kappa \kappa_{1} \kappa_{2}}^{j m n}=\left(k_{1} l_{2}-k_{2} l_{1}\right)\left(\kappa_{2}^{2}+a_{n}^{2}-\kappa_{1}^{2}-a_{m}^{2}\right)\left(\kappa^{2}+a_{j}^{2}\right)^{-1} .
$$

From the analysis in (Kozlov et al., 1987; Soomere, 1996) it follows that the two-layer model may contain a purely barotropic flow (that exists only owing to specific form of Eq. (2) and is unstable with respect to small baroclinic disturbances). It is also easy to show that, formally, a purely barotropic flow may exist in the three-layer model. Indeed, if at some time moment the energy of the first and the second baroclinic mode is zero, then

$$
\begin{aligned}
& \frac{\partial F_{j}\left(\tau_{0}\right)}{\partial \tau}=4 \pi I_{j 1 j}= \\
& =4 \pi \int D_{\kappa \kappa_{1} \kappa_{2}}^{j 1 j} K_{j 1 j} \delta\left(\omega_{j 1 j}^{012}\right) \delta\left(\kappa_{012}\right) d \kappa_{12}=0, j=2,3,
\end{aligned}
$$

and there is no energy flow into baroclinic modes although the coupling coefficients $\gamma_{12}^{2}$ and $\gamma_{13}^{3}$ are nonzero. The absence of the energy flow results from the structure of the kernel of the collision integrals $I_{j 1 j}$ that are proportional to the energy of the baroclinic mode. However, in a certain sense this asymmetry results from the fact that the coupling coefficients $\gamma_{11}^{2}$ and $\gamma_{11}^{3}$ vanish. Notice that analogical coefficients $\gamma_{11}^{j}$ vanish in the model with an arbitrary number of layers; thus, the asymmetry of the energy flow from the barotropic mode to the baroclinic modes is a general property of models of this type.

It is also interesting to notice that solely the barotropic mode may evolve uncoupled with other modes whereas a superposition of the barotropic and the first baroclinic mode may not. If at some time moment the barotropic and the first barotropic motions are present and only the second baroclinic 
Table 1. Conditions of vanishing of the coupling coefficients. Symbol "+" means that the corresponding coefficient never vanishes and " 0 " means that it is identically zero

\begin{tabular}{ccccc}
\hline Mode & Interaction with & Barotropic mode & $1^{\text {st }}$ baroclinic mode & $2^{\text {nd }}$ baroclinic mode \\
\hline Barotropic & Barotropic mode & + & 0 & 0 \\
& $1^{\text {st }}$ baroclinic mode & 0 & + & 0 \\
& $2^{\text {nd }}$ baroclinic mode & 0 & 0 & + \\
\hline $1^{\text {st }}$ baroclinic & Barotropic mode & 0 & + & 0 \\
& $1^{\text {st }}$ baroclinic mode & + & $h_{1} \delta \rho_{2}=h_{3} \delta \rho_{3} \& h_{1}=h_{3}$ & + \\
& $2^{\text {nd } \text { baroclinic mode }}$ & 0 & 0 & $h_{1} \delta \rho_{2}=h_{3} \delta \rho_{3}$ \\
\hline $2^{\text {nd }}$ baroclinic & Barotropic mode & 0 & + & + \\
& $1^{\text {st } \text { baroclinic mode }}$ & 0 & $h_{1} \delta \rho_{2}=h_{3} \delta \rho_{3}$ \\
& $2^{\text {nd }}$ baroclinic mode & + & $h_{1} \delta \rho_{2}=h_{3} \delta \rho_{3}$ & $h_{1} \delta \rho_{2}=h_{3} \delta \rho_{3} \& h_{2}=h_{1}+h_{3}$ \\
\hline
\end{tabular}

mode is void of energy, then

$$
\begin{aligned}
& \frac{\partial F_{3}}{\partial \tau}=\pi \int\left(\gamma_{22}^{3} C_{\kappa \kappa_{1} \kappa_{2}}^{322}\right)^{2} F_{2}\left(\kappa_{1}\right) F_{2}\left(\kappa_{2}\right) \times \\
& \times \delta\left(\omega_{322}^{012}\right) \delta\left(\kappa_{012}\right) d \kappa_{12} \geq 0
\end{aligned}
$$

because the coefficient $\gamma_{22}^{3}$ never vanishes.

\section{Discussion}

Although the kinetic equation has been derived for the particular system of Rossby waves in a multi-layer ocean, most of the analysis remains valid for any set of wave systems possessing mutual interaction described by the matrix $\mathbf{A}$ in Eq. (4). The basic properties of kinetic equations in the threelayer model are mostly direct generalisations of those of two-layer model. This equation possesses a fully barotropic solution in consistence with the possibility of the purely barotropic flow to exist in the framework of the governing equations. If energy is concentrated in the baroclinic modes, the barotropic mode will necessarily be generated. An important feature is that motions initially consisting of the barotropic and one baroclinic mode always transfer energy to the other baroclinic mode. Thus, a solution consisting of a superposition of the barotropic and the first baroclinic mode does not exist in the three-layer model.

Although a number of external parameters enter into Eq. (1), basic properties of a particular three-layer model only depend on four external parameters: density changes between the layers and the ratios of the depths of the layers. The structure of Eqs. (3) and (4) confirms the well-known fact that only changes of the vertical structure of the motion may alter the modal structure. Changes of other external parameters only lead to multiplication of all the entries of the matrix $\mathbf{A}$ in Eq. (4), its eigenvalues and eigenvectors by a certain factor. The above has shown that degeneration of certain coefficients only occurs, as it may be expected, in partially symmetric situations where $h_{1} \delta \rho_{2}=h_{3} \delta \rho_{3}$ (i.e. the reduced depths of the topmost and the lowest layers are equal). Addi- tional symmetries $h_{1}=h_{3}$ or $h_{2}=h_{1}+h_{3}$ cause vanishing of some other coefficients.

An interesting property is that the largest possible number of coupling coefficients vanish in the fairly realistic case when the density changes between layers are equal, depths of the topmost and the lowest layers are equal, and the intermediate layer is twice as thick as the other layers. This leads to a delicate situation where energy redistribution is governed by a few classes of interacting waves. The absence of a part of interactions may lead to basically different evolution scenarios of the whole system as suggested in (Soomere, 1995, 1996).

The analysis reveals that in the three-layer ocean model one class of interactions is implicitly neglected. Indeed, energy exchange within triplets consisting of waves from three different modes is idle since the corresponding coupling coefficient is zero. This property reflects the fact that the eigenvectors corresponding to the nonzero eigenvalues are orthogonal with respect to the remaining eigenvector. The physical reason is that the intensity of such interactions is of the order of the vertical density alteration (Kozlov et al., 1987). Thus, the three-layer model implicitly excludes a whole class of interactions and at least four-layer model should be introduced to obtain a comprehensive picture of energy exchange patterns due to various types of resonant interactions of Rossby waves.

\section{Appendix A The analytical expressions for coupling co- efficients}

In the simplest baroclinic two-layer case the matrix $\mathbf{A}$ in Eq. (4) is

$\mathbf{A}^{(2)}=\frac{f_{0}^{2} \rho_{0}}{g \delta \rho_{2}}\left\|\begin{array}{cc}-\frac{1}{h_{1}} & \frac{1}{h_{1}} \\ \frac{1}{h_{2}} & -\frac{1}{h_{2}}\end{array}\right\|$.

Its eigenvalues, eigenvectors, and matrices $\mathbf{S}, \mathbf{S}^{-1}$ can be explicitly calculated:

$\lambda_{1}=0, \lambda_{2}=-\frac{1}{h_{1}}-\frac{1}{h_{2}}$, 
$\mathbf{S}=\left\|\begin{array}{cc}a & b h_{2} \\ a & -b h_{1}\end{array}\right\|, \quad \mathbf{S}^{-1}=\frac{1}{h_{1}+h_{2}}\left\|\begin{array}{cc}\frac{h_{1}}{a} & \frac{h_{2}}{a} \\ \frac{-1}{b} & \frac{1}{b}\end{array}\right\|$.

Here $a, b$ are arbitrary nonzero constants. The above has shown that $\gamma_{11}^{1}=\gamma_{12}^{2}=a, \gamma_{12}^{1}=\gamma_{21}^{1}=0$ and $\gamma_{12}^{2}=\gamma_{21}^{2}$. It is straightforward to evaluate the remaining coefficients:

$\gamma_{22}^{1}=\frac{b^{2} h_{1} h_{2}}{a}, \gamma_{22}^{2}=b\left(h_{2}-h_{1}\right)$.

From Eqs. (16)-(19) it follows that only 8 of total 27 coupling coefficients in the three-layer model have to be evaluated. The matrix $\mathbf{A}$ obviously has a zero eigenvalue $\lambda_{1}=0$ because the sum of elements of each line is zero. Other eigenvalues satisfy the following relations

$\lambda_{2} \lambda_{3}=d_{1} d_{2}+d_{1} c_{3}+c_{2} c_{3}$

$\lambda_{2}+\lambda_{3}=-\left(d_{1}+d_{2}+c_{2}+c_{3}\right)$.

The components $s_{1}, s_{2}, s_{3}$ of the eigenvectors can be found from equations $\mathbf{A}-\lambda_{j} \mathbf{I}=\mathbf{0}, j=1,2,3$ :

$-s_{1}\left(\lambda+d_{1}\right)+d_{1} s_{2}=0$,

$c_{2} s_{1}-c_{2} s_{2}-d_{2} s_{2}-\lambda s_{2}+d_{2} s_{3}=0$,

$c_{3} s_{2}-\left(\lambda+c_{3}\right) s_{3}=0$.

The eigenvector corresponding to $\lambda_{1}=0$ consists of equal elements $s_{1}=s_{2}=s_{3}=a$ (here $a$ is an arbitrary constant) whereas the second equation of Eqs. (A3) is identically satisfied. In the case $\lambda_{j} \neq 0$ (i.e. $j=2,3$ ) Eq. (A3) yield

$s_{1} h_{1}+s_{2} h_{2}+s_{3} h_{3}=0$

Equation (A4) is a particular form of a general result saying that the eigenvectors of the matrix are orthogonal with respect to the weights $h_{1}, \ldots, h_{N}$ (Kamenkovich, 1986).

It is convenient to represent the eigenvectors $\boldsymbol{s}^{2}$ and $\boldsymbol{s}^{3}$ in terms of $\lambda_{j}, j=2,3$. The resulting transfer matrix $\mathbf{S}$ and its inverse are

$$
\begin{aligned}
& \mathbf{S}=\left\|\begin{array}{ccc}
a & b & c \\
a & b \frac{\lambda_{2}+d_{1}}{d_{1}} & c \frac{\lambda_{3}+d_{1}}{d_{1}} \\
a & b \frac{\left(\frac{\lambda 2}{2}+d_{1}\right) c_{3}}{\left(\lambda_{2}+c_{3}\right) d_{1}} & c \frac{\left(\lambda_{3}+d_{1}\right) c_{3}}{\left(\lambda_{3}+c_{3}\right) d_{1}}
\end{array}\right\|, \\
& \mathbf{S}^{-1}=\left\|\begin{array}{c}
\frac{d_{1} d_{2}}{a \lambda_{2} \lambda_{3}}\left(\frac{c_{2} c_{3}}{d_{1} d_{2}}, \frac{c_{3}}{d_{2}}, 1\right) \\
\frac{d_{1} d_{2}}{b \lambda_{2}\left(\lambda_{3}-\lambda_{2}\right)}\left(\frac{\lambda_{3}+d_{1}}{\lambda_{3}+c_{3}}, \frac{c_{3}-d_{1}}{\lambda_{3}+c_{3}},-1\right) \\
\frac{d_{1} d_{2}}{c \lambda_{3}\left(\lambda_{3}-\lambda_{2}\right)}\left(-\frac{\lambda_{2}+d_{1}}{\lambda_{2}+c_{3}},-\frac{c_{3}-d_{1}}{\lambda_{2}+c_{3}}, 1\right)
\end{array}\right\|,
\end{aligned}
$$

where $b, c$ are arbitrary nonzero constants. In the particular case of Rossby waves, the expression for $\hat{\mathbf{s}}_{1}$ reduces to $\hat{\mathbf{s}}_{1}=\left(h_{1}, h_{2}, h_{3}\right) /\left[a\left(h_{1}+h_{2}+h_{3}\right)\right]$ that is just a variation of Eq. (A4).

Among the coupling coefficients of the barotropic mode, only expressions for $\gamma_{22}^{1}$ and $\gamma_{33}^{1}$ have not been found yet. From Eqs. (16) and (A5) it follows that

$\gamma_{22}^{1}=\frac{b^{2}}{a} G_{1}\left(\lambda_{2}\right), \quad \gamma_{33}^{1}=\frac{c^{2}}{a} G_{1}\left(\lambda_{3}\right)$, where

$G_{1}\left(\lambda_{2}\right)=\frac{d_{2} c_{3}}{\lambda_{2} \lambda_{3}}\left[\frac{c_{2}}{d_{2}}+\frac{\left(\lambda_{2}+d_{1}\right)^{2}}{d_{1} d_{2}}+\frac{c_{3}\left(\lambda_{2}+d_{1}\right)^{2}}{d_{1}\left(\lambda_{2}+c_{3}\right)^{2}}\right]$.

It is convenient to subsequently simplify the expression for $G_{1}\left(\lambda_{2}\right)$. From Eq. (A2) it follows that

$\left(\lambda_{2}+c_{3}\right)\left(\lambda_{3}+c_{3}\right)=d_{2}\left(d_{1}-c_{3}\right)$,

$\left(\lambda_{2}+d_{1}\right)\left(\lambda_{3}+c_{3}\right)=\left(\lambda_{2}+d_{1}+c_{2}\right)\left(c_{3}-d_{1}\right)$.

With the use of Eq. (A7), the ratio in the third additive of $G_{1}\left(\lambda_{2}\right)$ can be evaluated as

$\frac{\lambda_{2}+d_{1}}{\lambda_{2}+c_{3}}=-\frac{\lambda_{2}+d_{1}+c_{2}}{d_{2}}$.

Substituting Eq. (A8) into Eq. (A6) and rearranging the additives yields

$G_{1}\left(\lambda_{2}\right)=\frac{c_{2} c_{3}}{\lambda_{2} \lambda_{3}}\left[1-\frac{c_{3}\left(\lambda_{2}+d_{1}\right)}{d_{1}\left(\lambda_{2}+c_{3}\right)}\right]+$

$+\frac{c_{3}\left(\lambda_{2}+d_{1}\right)^{2}}{d_{1} \lambda_{2} \lambda_{3}}\left(1-\frac{c_{3}}{\lambda_{2}+c_{3}}\right)$.

Simplification of the expressions in brackets of Eq. (A9) yields that $G_{1}\left(\lambda_{2}\right)$ contains the factor $\lambda_{2}$ and can be expressed as

$G_{1}\left(\lambda_{2}\right)=\frac{c_{3}}{d_{1} \lambda_{3}\left(\lambda_{2}+c_{3}\right)}\left[c_{2}\left(d_{1}-c_{3}\right)+\left(\lambda_{2}+d_{1}\right)^{2}\right]$.

Substituting the equality

$\left(\lambda_{2}+d_{1}\right)\left(\lambda_{3}+d_{1}\right)=c_{2}\left(c_{3}-d_{1}\right)$

into Eq. (A9) reveals that

$G_{1}\left(\lambda_{2}\right)=\frac{c_{3}\left(\lambda_{2}-\lambda_{3}\right)\left(\lambda_{2}+d_{1}\right)}{\lambda_{3} d_{1}\left(\lambda_{2}+c_{3}\right)}$,

$\gamma_{22}^{1}=\frac{b^{2}\left(\lambda_{2}-\lambda_{3}\right)\left(\lambda_{2}+d_{1}\right) c_{3}}{a \lambda_{2}\left(\lambda_{3}+c_{3}\right) d_{1}}$.

$\gamma_{33}^{1}=\frac{c^{2}\left(\lambda_{3}-\lambda_{2}\right)\left(\lambda_{3}+d_{1}\right) c_{3}}{a \lambda_{2}\left(\lambda_{3}+c_{3}\right) d_{1}}$.

The coefficients $\gamma_{23}^{2}, \gamma_{33}^{2}, \gamma_{22}^{3}$ and $\gamma_{23}^{3}$ are:

$\gamma_{23}^{2}=\frac{c d_{1} d_{2}\left(\lambda_{3}+d_{1}\right)}{\lambda_{2}\left(\lambda_{3}-\lambda_{2}\right)\left(\lambda_{3}+c_{3}\right)} G_{2}\left(\lambda_{2}\right)$,

$\gamma_{33}^{2}=\frac{c^{2} d_{1} d_{2}\left(\lambda_{3}+d_{1}\right)}{b \lambda_{2}\left(\lambda_{3}-\lambda_{2}\right)\left(\lambda_{3}+c_{3}\right)} G_{2}\left(\lambda_{3}\right)$,

$\gamma_{22}^{3}=-\frac{b^{2} d_{1} d_{2}\left(\lambda_{2}+d_{1}\right)}{c \lambda_{3}\left(\lambda_{3}-\lambda_{2}\right)\left(\lambda_{2}+c_{3}\right)} G_{2}\left(\lambda_{2}\right)$,

$\gamma_{23}^{3}=-\frac{b d_{1} d_{2}\left(\lambda_{2}+d_{1}\right)}{\lambda_{3}\left(\lambda_{3}-\lambda_{2}\right)\left(\lambda_{2}+c_{3}\right)} G_{2}\left(\lambda_{3}\right)$,

where

$G_{2}\left(\lambda_{2}\right)=1+\frac{\left(\lambda_{2}+d_{1}\right)\left(c_{3}-d_{1}\right)}{d_{1}^{2}}-\frac{\left(\lambda_{2}+d_{1}\right) c_{3}^{2}}{\left(\lambda_{2}+c_{3}\right) d_{1}^{2}}$. 
Splitting the second additive in Eq. (A12) and regrouping the terms gives

$G_{2}\left(\lambda_{2}\right)=1-\frac{\left(\lambda_{2}+d_{1}\right)}{d_{1}}+\frac{c_{3}\left(\lambda_{2}+d_{1}\right)}{d_{1}^{2}}\left(1-\frac{c_{3}}{\lambda_{2}+c_{3}}\right)=$ $=\frac{\lambda_{2}}{d_{1}}\left[\frac{c_{3}\left(\lambda_{2}+d_{1}\right)}{d_{1}\left(\lambda_{2}+c_{3}\right)}-1\right]$.

Making use of Eq. (A7) yields

$G_{2}\left(\lambda_{2}\right)=\frac{\lambda_{2}^{2}\left(c_{3}-d_{1}\right)}{d_{1}^{2}\left(\lambda_{2}+c_{3}\right)}=\frac{\lambda_{2}^{2}\left(\lambda_{3}+c_{3}\right)}{d_{1}^{2} d_{2}}$,

$\gamma_{23}^{2}=\frac{c \lambda_{2}\left(\lambda_{3}+d_{1}\right)}{\left(\lambda_{3}-\lambda_{2}\right) d_{1}}, \gamma_{23}^{3}=-\frac{b \lambda_{3}\left(\lambda_{2}+d_{1}\right)}{\left(\lambda_{3}-\lambda_{2}\right) d_{1}}$,

$\gamma_{33}^{2}=\frac{c^{2} \lambda_{3}^{2}\left(\lambda_{3}+d_{1}\right)\left(\lambda_{2}+c_{3}\right)}{b \lambda_{2}\left(\lambda_{3}-\lambda_{2}\right)\left(\lambda_{3}+c_{3}\right) d_{1}}$,

$\gamma_{22}^{3}=-\frac{b^{2} \lambda_{2}^{2}\left(\lambda_{2}+d_{1}\right)\left(\lambda_{3}+c_{3}\right)}{c \lambda_{3}\left(\lambda_{3}-\lambda_{2}\right)\left(\lambda_{2}+c_{3}\right) d_{1}}$.

The remaining coefficients $\gamma_{22}^{2}$ and $\gamma_{33}^{3}$ do not have such a simple multiplicative form as other coefficients. They can be expressed as

$\gamma_{22}^{2}=\frac{b d_{1} d_{2}}{\lambda_{2}\left(\lambda_{3}-\lambda_{2}\right)} G_{3}\left(\lambda_{2}, \lambda_{3}\right)$,

$\gamma_{33}^{3}=-\frac{c d_{1} d_{2}}{\lambda_{3}\left(\lambda_{3}-\lambda_{2}\right)} G_{3}\left(\lambda_{3}, \lambda_{2}\right)$,

where

$G_{3}\left(\lambda_{2}, \lambda_{3}\right)=$

$=\frac{\lambda_{3}+d_{1}}{\lambda_{3}+c_{3}}+\frac{\left(c_{3}-d_{1}\right)\left(\lambda_{2}+d_{1}\right)^{2}}{d_{1}^{2}\left(\lambda_{3}+c_{3}\right)}-\frac{\left(\lambda_{2}+d_{1}\right)^{2} c_{3}^{2}}{d_{1}^{2}\left(\lambda_{2}+c_{3}\right)^{2}}$.

It is convenient to split the second additive in Eq. (A15) into two parts. After regrouping the additives, Eq. (A14) can be reduced to

$$
\begin{aligned}
& G_{3}\left(\lambda_{2}, \lambda_{3}\right)=\frac{1}{\left(\lambda_{3}+c_{3}\right)}\left[\left(\lambda_{3}+d_{1}\right)-\frac{\left(\lambda_{2}+d_{1}\right)^{2}}{d_{1}}\right]+ \\
& +\frac{\left(\lambda_{2}+d_{1}\right)^{2} c_{3}}{d_{1} d_{1}}\left[\frac{1}{\left(\lambda_{3}+c_{3}\right)}-\frac{c_{3}}{\left(\lambda_{2}+c_{3}\right)}\right] .
\end{aligned}
$$

Simplification of the expressions in the square brackets yields

$$
\begin{aligned}
& G_{3}\left(\lambda_{2}, \lambda_{3}\right)=\frac{\lambda_{2}-\lambda_{3}}{\lambda_{3}+c_{3}}\left[\frac{c_{3}^{2}\left(\lambda_{2}+d_{1}\right)^{2}}{d_{1}^{2}\left(\lambda_{2}+c_{3}\right)^{2}}-1\right]+ \\
& +\frac{\lambda_{2}\left(\lambda_{2}+d_{1}\right)}{\left(\lambda_{3}+c_{3}\right) d_{1}}\left[\frac{c_{3}\left(\lambda_{2}+d_{1}\right)}{d_{1}\left(\lambda_{2}+c_{3}\right)}-1\right] .
\end{aligned}
$$

From Eq. (A16) it follows that $G_{3}\left(\lambda_{2}, \lambda_{3}\right)$ contains the explicit factor $\lambda_{2}\left(c_{3}-d_{1}\right)$. With the use of Eq. (A10), Eqs. (A14) and (A16) can be reduced to

$$
\begin{aligned}
& G_{3}\left(\lambda_{2}, \lambda_{3}\right)= \\
& =\frac{\lambda_{2}}{d_{1} d_{2}}\left\{\left(\lambda_{3}-\lambda_{2}\right)\left[\frac{c_{3}\left(\lambda_{2}+d_{1}\right)}{d_{1}\left(\lambda_{2}+c_{3}\right)}+1\right]-\frac{\lambda_{2}\left(\lambda_{2}+d_{1}\right)}{d_{1}}\right\},
\end{aligned}
$$

$$
\begin{aligned}
& \gamma_{22}^{2}=b\left[\frac{c_{3}\left(\lambda_{2}+d_{1}\right)}{d_{1}\left(\lambda_{2}+c_{3}\right)}+1-\frac{\lambda_{2}\left(\lambda_{2}+d_{1}\right)}{\left(\lambda_{3}-\lambda_{2}\right) d_{1}}\right], \\
& \gamma_{33}^{3}=c\left[\frac{c_{3}\left(\lambda_{3}+d_{1}\right)}{d_{1}\left(\lambda_{3}+c_{3}\right)}+1+\frac{\lambda_{3}\left(\lambda_{3}+d_{1}\right)}{\left(\lambda_{3}-\lambda_{2}\right) d_{1}}\right] .
\end{aligned}
$$

The expressions for the coupling coefficients are much simpler in the particular case $c_{1}=d_{3}$ and can be obtained in a straightforward way from the following representation of the transfer matrix $\mathbf{S}$ and its inverse:

$$
\begin{aligned}
& \mathbf{S}=\left\|\begin{array}{ccc}
a & b & c \\
a & 0 & -\frac{c\left(d_{2}+c_{2}\right)}{d_{1}} \\
a & -\frac{b c_{2}}{d_{2}} & c
\end{array}\right\|, \\
& \mathbf{S}^{-1}=\left\|\begin{array}{c}
\frac{1}{a\left(d_{1}+d_{2}+c_{2}\right)}\left(c_{2}, d_{1}, d_{2}\right) \\
\frac{d_{2}}{b\left(d_{2}+c_{2}\right)}(1,0,-1) \\
\frac{d_{1}}{c\left(d_{1}+d_{2}+c_{2}\right)}\left(\frac{c_{2}}{d_{2}+c_{2}},-1, \frac{d_{2}}{d_{2}+c_{2}}\right)
\end{array}\right\| .
\end{aligned}
$$

Acknowledgements. The study has been supported by Estonian Science Foundation, Grant No 4025. J. Laanemets and M.-J. Lilover kindly presented density data from the Gotland Basin, Baltic Sea.

\section{References}

Aitsam, A., Hansen, H. P., Elken, J., Kahru, M., Laanemets, J., Pajuste, M., Pavelson, J., and Talpsepp, L.: Physical and chemical variability of the Baltic Sea: a joint experiment in the Gotland Basin, Cont. Shelf Res., 3, 291-310, 1984.

Axelsson, P.: Three-wave coupling in a stratified MHD plasma, Nonl. Proc. Geophys., 5, 105-110, 1998.

Balk, A. M., Zakharov, V. E., and Nazarenko, S. M.: On non-local turbulence of the drift waves, Soviet Phys., JETP, 71, 249-260, 1990.

Benney, D. J. and Newell, A. C.: Random wave closures, Stud. Appl. Math., 48, 29-53, 1969.

Carnevale, G. F.: Statistical features of the evolution of twodimensional turbulence, J. Fluid Mech., 122, 143-153, 1982.

Hasselmann, K.: On the nonlinear energy transfer in a gravity-wave spectrum. Part 1. General theory, J. Fluid Mech., 12, 481-500, 1962.

Jones, S.: Rossby wave interactions and instabilities in a rotating, two-layer fluid on a beta-plane. Part I: Resonant interactions, Geophys. Astrophys. Fluid Dyn., 11, 289-332, 1979.

Kamenkovich, V. M., Koschlyakov, V. N., and Monin, A. S.: Synoptic eddies in the ocean, Reidel, Dordrecht, Holland, 1986.

Kenyon, K.: Nonlinear energy transfer in a Rossby-wave spectrum, in Notes on the 1964 Summer Study Program in Geophysical Fluid Dynamics at the Woods Hole Oceanographic Institute, Student lectures, 11, 69-83, 1964.

Komen, G. J., Cavaleri, L., Donelan, M., Hasselmann, K., Hasselmann, S., and Janssen, P. A. E. M.: Dynamics and modelling of ocean waves, Cambridge University Press, Cambridge, 1994.

Kozlov, O. V., Reznik, G. M., and Soomere, T.: Weak turbulence on the $\beta$-plane in a two-layer ocean, Izv. Acad. Sci. USSR. Atm. Oceanic Phys., 23, 869-874, 1987.

Longuet-Higgins, M. S. and Gill, A. E.: Resonant interactions between planetary waves, Phil. Trans. Roy. Soc. Lond., 294, 120 140, 1967.

Majda, A. J., McLaughlin, D. W., and Tabak, E. G.: A onedimensional model for dispersive wave turbulence, J. Nonlinear Sci., 7, 9-44, 1997. 
Piterbarg, L. I.: Hamiltonian formalism for Rossby waves, in: Nonlinear waves and weak turbulence, (Ed.) Zakharov, V. E., Amer. Math. Soc. Transl. (2), 182, 131-166, 1998.

Reznik, G. M.: On the energy transfer equation for weakly interacting waves, Int. J. Nonlin. Mech., 19, 95-113, 1984.

Reznik, G. M.: Weak turbulence on a $\beta$-plane, in: Synoptic eddies in the ocean, (Eds.) Kamenkovich, V. M., Koschlyakov, V. N., and Monin, A. S., 73-108, Reidel, Dordrecht, Holland, 1986.

Soomere, T.: Generation of zonal flow and meridional anisotropy in two-layer weak geostrophic turbulence, Phys. Rev. Lett., 75, 2440-2443, 1995.

Soomere, T.: Spectral evolution of two-layer weak geostrophic turbulence. Part I: Typical scenarios, Nonl. Proc. Geophys., 3, 166195, 1996.
Soomere, T.: New insight into classical equilibrium solutions of kinetic equations, J. Nonlinear Sci., 11, 305-320, 2001.

Vallis, G. K. and Maltrud M. E.: Generation of mean flows and jets on a beta plane and over topography, J. Phys. Oceanogr., 23, 1346-1362, 1993.

Wiklund, K.: Wave interactions in a shallow-water model, Nonl. Proc. Geophys., 5, 137-144, 1999.

Zakharov, V. E., L'vov, V. S., and Falkovich, G.: Kolmogorov spectra of turbulence I: Wave turbulence, Springer, Berlin, Heidelberg, New York, 1992.

Zakharov, V. E., and Pushkarev, A. N.: Diffusion model of interacting gravity waves on the surface of deep fluid,Nonl. Proc. Geophys., 6, 1-10, 1999. 